Original article

\title{
Characteristics of adult patients with chronic intestinal failure due to short bowel syndrome: An international multicenter survey
}

Loris Pironi ab, *, Ezra Steiger ${ }^{c}$, Francisca Joly ${ }^{\mathrm{d}}$, Palle B. Jeppesen ${ }^{\mathrm{e}}$, Geert Wanten ${ }^{\mathrm{f}}$, Anna S. Sasdelli ${ }^{\mathrm{b}}$, Cecile Chambrier ${ }^{\mathrm{g}}$, Umberto Aimasso ${ }^{\mathrm{h}}$, Manpreet M. Mundi ${ }^{\mathrm{i}}$, Kinga Szczepanek ${ }^{\mathrm{j}}$, Amelia Jukes ${ }^{\mathrm{k}}$, Miriam Theilla ${ }^{1}$, Marek Kunecki ${ }^{\mathrm{m}}$, Joanne Daniels ${ }^{\mathrm{n}}$, Mireille Serlie $^{\circ}$, Florian Poullenot ${ }^{\mathrm{p}}$, Sheldon C. Cooper ${ }^{\mathrm{q}}$, Henrik H. Rasmussen ${ }^{\mathrm{r}}$, Charlene Compher ${ }^{\mathrm{s}}$, David Seguy ${ }^{\mathrm{t}}$, Adriana Crivelli ${ }^{\mathrm{u}}$, Lidia Santarpia ${ }^{\mathrm{v}}$, Francesco W. Guglielmi w, Nada Rotovnik Kozjek ${ }^{x}$, Stéphane M. Schneider ${ }^{\text {, }}$, Lars Ellegard $^{\mathrm{z}}$, Ronan Thibault ${ }^{\mathrm{ab}}$, Przemysław Matras ${ }^{\mathrm{ac}}$, Konrad Matysiak ad, Andrè Van Gossum ${ }^{\text {ae }}$, Alastair Forbes ${ }^{\text {af }}$, Nicola Wyer ${ }^{\text {ag }}$, Marina Taus ${ }^{\text {ah }}$, Nuria M. Virgili ai, Margie O'Callaghan aj, Brooke Chapman ${ }^{\text {ak }}$, Emma Osland ${ }^{\text {al }}$, Cristina Cuerda am, Gábor Udvarhelyi an ${ }^{\text {, Lynn Jones }}{ }^{\text {ao }}$, Andre D. Won Lee ${ }^{\text {ap }}$, Luisa Masconale ${ }^{\text {aq }}$, Paolo Orlandoni ${ }^{\text {ar }}$, Corrado Spaggiari ${ }^{\text {as }}$, Marta Bueno Díez ${ }^{\text {at }}$, Maryana Doitchinova-Simeonova ${ }^{a u}$, Aurora E. Serralde-Zúñiga ${ }^{a v}$, Gabriel Olveira ${ }^{\text {aw }}$, Zeljko Krznaric ${ }^{a x}$, Laszlo Czako ${ }^{\text {ay }}$, Gintautas Kekstas az, Alejandro Sanz-Paris ba, $\mathrm{M}^{\mathrm{a}}$ Estrella Petrina Jáuregui ${ }^{\mathrm{bb}}$, Ana Zugasti Murillo ${ }^{\mathrm{bc}}$, Eszter Schafer ${ }^{\text {bd }}$, Jann Arends be, José P. Suárez-Llanos bf , Nader N. Youssef ${ }^{\text {bg }}$, Giorgia Brillanti a , Elena Nardi ${ }^{\text {bh }}$, Simon Lal ${ }^{\text {bi }}$, The Home Artificial Nutrition and Chronic Intestinal Failure Special Interest Group of ESPEN, The European Society for Clinical Nutrition and Metabolism

\footnotetext{
a Alma Mater Studiorum, University of Bologna, Department of Medical and Surgical Sciences, Bologna, Italy

${ }^{\mathrm{b}}$ IRCCS Azienda Ospedaliero-Universitaria di Bologna, Centre for Chronic Intestinal Failure - Clinical Nutrition and Metabolism Unit, Bologna, Italy

${ }^{\mathrm{c}}$ Home Nutrition Support, Cleveland Clinic Foundation, Cleveland, OH, USA

d Centre for Intestinal Failure, Department of Gastroenterology and Nutritional Support, Hôpital Beaujon, Clichy, France

e Rigshospitalet, Department of Gastroenterology, Copenhagen, Denmark

${ }^{\mathrm{f}}$ Intestinal Failure Unit, Department of Gastroenterology and Hepatology, Radboud University Nijmegen Medical Centre, Nijmegen, the Netherlands

${ }^{g}$ Unité de Nutrition Clinique Intensive, Hospices Civils de Lyon, Hôpital Lyon Sud, Lyon, France

h Città Della Salute e Della Scienza, Turin, Italy

${ }^{i}$ Division of Gastroenterology and Hepatology, Mayo Clinic College of Medicine, Rochester, MN, USA

j General and Oncology Surgery Unit, Stanley Dudrick's Memorial Hospital, Skawina, Poland

${ }^{\mathrm{k}}$ University Hospital of Wales, Cardiff, United Kingdom

${ }^{1}$ Rabin Medical Center, Petach Tikva, Sackler School of Medicine, Tel Aviv University, Israel

${ }^{\mathrm{m}}$ M. Pirogow Hospital, Lodz, Poland

${ }^{n}$ Nottingham University Hospital NHS Trust, Nottingham, United Kingdom

${ }^{\circ}$ Amsterdam University Medical Center, Amsterdam, the Netherlands

p Service de Gastroentérologie, Hôpital Haut-Lévêque, CHU de Bordeaux, Pessac, France

${ }^{\mathrm{q}}$ University Hospitals Birmingham NHS Foundation Trust, Birmingham, United Kingdom

${ }^{\mathrm{r}}$ Centre for Nutrition and Bowel Disease, Department of Gastroenterology, Aalborg University Hospital, Aalborg, Denmark

${ }^{s}$ Hospital of the University of Pennsylvania, University of Pennsylvania School of Nursing, Philadelphia, PA, USA

${ }^{\mathrm{t}}$ Service de Nutrition, CHRU de Lille, Lille, France

u Unidad de Soporte Nutricional, Rehabilitación y Trasplante de Intestino, Hospital Universitario Fundacion Favaloro, Buenos Aires, Argentina

${ }^{\mathrm{V}}$ Federico II University, Naples, Italy

${ }^{\mathrm{w}}$ Gastroenterology Unit, Monsignor di Miccoli Hospital, Barletta, Italy

${ }^{\mathrm{x}}$ Institute of Oncology, Ljubljana, Slovenia

${ }^{y}$ Gastroenterology and Clinical Nutrition, CHU of Nice, Université Côte D'Azur, Nice, France
}

* Corresponding author. IRCCS Azienda Ospedaliero-Universitaria di Bologna, Centre for Chronic Intestinal Failure - Clinical Nutrition and Metabolism Unit, Department of Medical and Surgical Sciences, University of Bologna, Italy. 
${ }^{z}$ Department of Internal Medicine and Clinical Nutrition, Institute of Medicine, Sahlgrenska University Hospital, University of Gothenburg, Gothenburg, Sweden

${ }^{\mathrm{ab}}$ CHU Rennes, Nutrition Unit, Clinique Saint Yves, Home Parenteral Nutrition Centre, INRAE, INSERM, Univ Rennes, Nutrition Metabolisms and Cancer, NuMeCan, Rennes, France

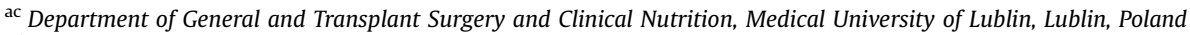

${ }^{\text {ad }}$ Centre for Intestinal Failure, Department of General, Endocrinological and Gastroenterological Surgery, Poznań University of Medical Science, Poznań, Poland

ae Medico-Surgical Department of Gastroenterology, Hôpital Erasme, Free University of Brussels, Belgium

af Institute of Internal Medicine, University of Tartu, Tartu, Estonia, And Previously at Norfolk and Norwich University Hospital, Norwich, United Kingdom

ag University Hospitals Coventry \& Warwickshire NHS Trust, Coventry, United Kingdom

${ }^{\text {ah }}$ SOD Dietetica e Nutrizione Clinica, Centro Riferimento Regionale NAD, Ospedali Riuniti di Ancona, Italy

ai Facultatiu Especialista. Servei D'Endocrinologia I Nutrició, Hospital Universitari de Bellvitge, Barcelona, Spain

aj Flinders Medical Centre, Adelaide, Australia

${ }^{\mathrm{ak}}$ Austin Health, Melbourne, Australia

al Royal Brisbane and Women's Hospital, Herston, Australia

am Nutrition Unit, Hospital General Universitario Gregorio Marañon, Madrid, Spain

an St. Imre Hospital, Budapest, Hungary

ao Royal Prince Alfred Hospital, Camperdown, Australia

ap Hospital Das Clinicas da Faculdade de Medicina da Universidade de São Paulo, São Paulo, Brazil

aq Ospedale Orlandi, Bussolengo, VR, Italy

${ }^{\text {ar }}$ Nutrizione Clinica-Centro di Riferimento Regionale NAD, IRCCS-INRCA, Ancona, Italy

as AUSL Parma, Parma, Italy

at Servei D'Endocrinologia I Nutrició, Hospital Universitari Arnau de Vilanova, Lleida, Spain

au Bulgarian Executive Agency of Transplantation, Sofia, Bulgaria

av Instituto Nacional de Ciencias Médicas y Nutrición, Salvador Zubirán, Mexico, Mexico

${ }^{a w}$ Hospital Regional Universitario de Málaga, Málaga, Spain

${ }^{a x}$ Centre of Clinical Nutrition, Department of Medicine, University Hospital Centre, Zagreb, Croatia

ay First Department of Internal Medicine, Szeged, Hungary

${ }^{a z}$ Vilnius University Hospital Santariskiu Clinics, Vilnius, Lithuania

ba Miguel Servet Hospital, Zaragoza, Spain

bb Complejo Hospitalario de Navarra, Pamplona, Spain

bc Hospital Virgen Del Camino, Pamplona, Spain

bd Magyar Honvedseg Egészségügyi Központ (MHEK), Budapest, Hungary

be Department of Medicine, Oncology and Hematology, University of Freiburg, Germany

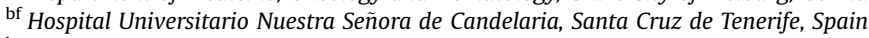

bg VectivBio AG Basel, Switzerland, Digestive Healthcare Center, NJ, USA

bh Department of Experimental, Diagnostic and Specialty Medicine, University of Bologna, Italy

bi Intestinal Failure Unit, Salford Royal Foundation Trust, Salford, UK

\section{A R T I C L E I N F O}

Article history:

Received 30 June 2021

Accepted 8 July 2021

\section{Keywords:}

Short bowel syndrome

Intestinal failure

Intravenous supplementation

Home parenteral nutrition

Epidemiology

\section{S U M M A R Y}

Background and aims: The case-mix of patients with intestinal failure due to short bowel syndrome (SBSIF) can differ among centres and may also be affected by the timeframe of data collection. Therefore, the ESPEN international multicenter cross-sectional survey was analyzed to compare the characteristics of SBS-IF cohorts collected within the same timeframe in different countries.

Methods: The study included 1880 adult SBS-IF patients collected in 2015 by 65 centres from 22 countries. The demographic, nutritional, SBS type (end jejunostomy, SBS-J; jejuno-colic anastomosis, SBS-JC; jejunoileal anastomosis with an intact colon and ileocecal valve, SBS-JIC), underlying disease and intravenous supplementation (IVS) characteristics were analyzed. IVS was classified as fluid and electrolyte alone (FE) or parenteral nutrition admixture (PN). The mean daily IVS volume, calculated on a weekly basis, was categorized as $<1,1-2,2-3$ and $>3 \mathrm{~L} /$ day.

Results: In the entire group: $60.7 \%$ were females and SBS-J comprised $60 \%$ of cases, while mesenteric ischaemia (MI) and Crohn' disease (CD) were the main underlying diseases. IVS dependency was longer than 3 years in around $50 \%$ of cases; IVS was infused $\geq 5$ days/week in $75 \%$ and FE in $10 \%$ of cases. Within the SBS-IF cohort: CD was twice and thrice more frequent in SBS-J than SBS-JC and SBS-JIC, respectively, while MI was more frequent in SBS-JC and SBS-JIC. Within countries: SBS-J represented $75 \%$ or more of patients in UK and Denmark and 50-60\% in the other countries, except Poland where SBS-JC prevailed. CD was the main underlying disease in UK, USA, Denmark and The Netherlands, while MI prevailed in France, Italy and Poland.

Conclusions: SBS-IF type is primarily determined by the underlying disease, with significant variation between countries. These novel data will be useful for planning and managing both clinical activity and research studies on SBS.

( $) 2021$ European Society for Clinical Nutrition and Metabolism. Published by Elsevier Ltd. All rights reserved.

\section{Introduction}

In adults, short bowel syndrome (SBS) occurs due to extensive surgical resection leaving less than $200 \mathrm{~cm}$ of small intestine, measured from the ligament of Treitz [1,2]. Patients typically present with diarrhea, malnutrition and dehydration [1,2]. In some patients, SBS can occur despite a post-resection small intestine length $>200 \mathrm{~cm}$ due to impairment of remnant bowel function 
(functional SBS) [1-3]. SBS is the main cause of chronic intestinal failure (CIF), which is defined as "the persistent reduction of the gut function below the minimum necessary for the absorption of macronutrients and/or water and electrolytes, such that intravenous supplementation (IVS) is required to maintain health and/or growth" [4]. For patients with CIF, IVS is provided at patient's home through home parenteral nutrition (HPN) programs [4]. The international multicenter cross-sectional survey carried out by the European Society for Clinical Nutrition and Metabolism (ESPEN), demonstrated that around two thirds of all CIF cases resulted from SBS (SBS-IF) [3].

Individual centre surveys have shown that the case-mix of patients with SBS-IF can differ between IF centres. Mesenteric ischemia (MI) and Crohn's disease (CD) accounted for 58.0\% (MI 20.5\%, CD 37.5\%) of the Salford cohort in the UK [5], 59.4\% (MI 35.0\%, CD 24.40\%) of the Copenhagen cohort in Denmark [6], 49.0\% (MI 43.0\%, CD 6.0\%) of the Paris cohort in France [7] and 53.1\% (MI 29.1\%, CD 24.0\%) of the Bologna cohort in Italy [8]. Also, the type of SBS differed among centres, with ranges of $18-82 \%$ for SBS-J, $6.2-67 \%$ for SBS-JC and 1.1-15\% for SBS-JIC [5-8]. However, the study design and the time-frame of data collection varied amongst these reports and could have contributed to the observed differences. The British, Danish and Italian studies were cross-sectional observations of patients who were on HPN in 2017, 2017 and 2020, respectively $[5,6,8]$. The French study included all patients with SBS-IF treated from 1980 to 2006 [7]. Despite these findings, it is currently unknown if these SBS-IF patient characteristics are comparable in other countries and/or whether they have remained consistent over time. Therefore, the SBS-IF cohort of the ESPEN international multicenter cross-sectional survey [3] was analyzed in order to compare patient characteristics at the same time in different countries.

\section{Materials and methods}

\subsection{Data collection and patient population}

The ESPEN international cross-sectional observational study on CIF [3] was the baseline of a prospective survey developed by the Home Artificial Nutrition and Chronic Intestinal Failure (HAN\&CIF) special interest group of ESPEN, aimed at investigating the outcome of patients with CIF [9]. Invitation to participate in the study occurred via representatives of the national Parenteral and Enteral Nutrition (PEN) societies of the ESPEN Council, who were asked to send the study protocol to members of their PEN societies. Sixtyfive HPN centers from 22 countries were interested in participating in the study and enrolled all adult patients ( $\geq 18$-year-old) who were dependent on HPN for either benign-CIF or malignantCIF on March 1st, 2015 [3]. The term malignant-CIF indicates the presence of active malignant disease [3]. Data were collected in a structured questionnaire embedded in an Excel (Microsoft Co., 2013) database, termed "the CIF Action day" [3,9]. Demographic, clinical, CIF, underlying disease, IVS and HPN program characteristics were collected. A total of 3362 patients were enrolled: 320 with malignant CIF and 2919 with benign CIF. The mechanisms of benign CIF were SBS-IF in $64.3 \%$, enterocutaneous fistulas in $7.0 \%$, intestinal dysmotility in $17.5 \%$, mechanical obstruction in $4.4 \%$ and extensive mucosal disease in 6.8\% [3]. For the purpose of the present study, only patients with benign SBS-IF were analyzed.

\subsection{Ethical statement}

The research was based on anonymized information taken from patient records at the time of data collection. The study was conducted with full regard to confidentiality of the individual patient.
Ethical committee approval was obtained by the individual HPN centers according to local regulations.

\subsection{Statistical analysis}

Depending on the anatomy of the remnant bowel, SBS was categorized as: end jejunostomy (SBS-J); jejuno-colic anastomosis (SBS-JC); jejunoileal anastomosis with an intact colon and ileocecal valve (SBS-JIC) [2,3]. Patients who required IVS because of a high output ileostomy were defined "functional SBS" and were included in the SBS-J group. The severity classification of CIF was based on the type and volume of IVS, calculated as daily mean of the total volume infused per week and consisted of eight categories: volume per day of infusion $\times$ number of infusions per week/7 (mL/day): FE1 or PN1, $\leq 1000$; FE2 or PN2, 1001-2000; FE3 or PN3, 2001-3000; FE4 or PN4, $>3000$ [9]. The daily mean volume and energy of IVS were calculated as follows: daily total volume ( $\mathrm{mL} /$ day) or energy $(\mathrm{kcal} /$ day $)=$ amount per day of infusion $\mathrm{x}$ number of infusions per week/7; daily volume or energy per kg of patient body weight $(\mathrm{mL} /$ $\mathrm{kgBW} /$ day or $\mathrm{kcal} / \mathrm{kgBW} /$ day) = amount per day of infusion $\mathrm{x}$ number of infusions per week)/7/kg patient body weight. The patient's body mass index (BMI) was calculated by Quetelet's formula (weight $(\mathrm{kg}) /$ height $\left(\mathrm{m}^{2}\right)$.

Data are reported as mean \pm standard deviation $\left(m_{ \pm} S D\right)$, minimum-maximum range and percentage.

The IBM SSPS Statistics package for Windows, version 23.0 (BM Co., Armonk, NY, USA) was used for the analyses.

\section{Results}

\subsection{Total population}

The total cohort consisted of 1880 SBS-IF patients, included by 65 centres from 22 countries: 1559 patients (82.9\%) were from European countries, the remainder were from the USA (n. 221, 11.8\%), Israel (n.21, 1.1\%), South and Central America (n. 35, 1.9\%) and Oceania (n.44, 3.3\%); 7 countries (Denmark, France, Italy, Poland, The Netherlands, UK, USA) enrolled more than 100 patients and 4 countries (Australia, Argentina, Spain, and Israel) each enrolled between 20 and 40 patients.

Table 1 shows the characteristics of the total group and of the gender cohorts.

Around two-thirds of patients were females (60.7\%), aged over 50 years (69\%) and mostly with a BMI within the normal range (61.9\%).

The SBS types were SBS-J in 1127 patients (40 of whom had a functional SBS), SBS-JC in 581 and SBS JIC in 172. Three-fourths of the underlying diseases were represented by $\mathrm{CD}$, MI, post-surgical complications, and radiation enteritis. CD and MI equally contributed to the majority (53.8\%) of total cases.

The duration of IVS was less than 3 years in around one-half of patients and longer than 10 years in one-sixth. Three-fourths of patients received IVS infusion 5 or more days per week (7 days in more than one-half). The type of IVS was FE in around $10 \%$ of patients, PN $<2$ L/day in one-half and PN $>2$ L/day in one-third.

The most evident differences between the gender cohorts were in body height and weight, shorter and lower in females, as expected, and in radiation enteritis as underlying disease, which was more frequent in females.

\subsection{Characteristics of the SBS type cohorts}

Patient characteristics are summarized in Fig. 1 and extensively reported in supplementary table 1. 
Table 1

Demographic nutritional status, intravenous supplementation, anatomical characteristics, and underlying diseases of patients with chronic intestinal failure due to short bowel syndrome.

\begin{tabular}{|c|c|c|c|}
\hline & Total n. 1880 & Males n. 738 & Females n. 1142 \\
\hline Age, $y r .(m \pm S D)$ & $56.7 \pm 15.3$ & $55.5 \pm 15.6$ & $57.5 \pm 15.0$ \\
\hline range & $18.0-92.0$ & $18.0-88.0$ & $19.0-92.0$ \\
\hline \multicolumn{4}{|l|}{ Age-class, yr (\%) } \\
\hline$\leq 29$ & 5.7 & 7.1 & 4.7 \\
\hline $30-49$ & 25.3 & 26.4 & 24.4 \\
\hline $50-69$ & 46.9 & 47.1 & 46.8 \\
\hline$\geq 70$ & 22.1 & 19.4 & 24.0 \\
\hline Body weight, $\mathrm{kg}(m \pm S D)$ & $62.8 \pm 13.8$ & $69.5 \pm 12.9$ & $58.5 \pm 12.7$ \\
\hline range & $24.5-119.0$ & $30.0-110.9$ & $24.5-119.0$ \\
\hline \multicolumn{4}{|l|}{ Body weight-class, $k g(\%)$} \\
\hline$<40$ & 1.9 & 0.8 & 2.5 \\
\hline $40-49$ & 14.8 & 4.6 & 21.5 \\
\hline $50-59$ & 28.0 & 15.9 & 35.8 \\
\hline $60-69$ & 26.8 & 30.8 & 24.2 \\
\hline $70-79$ & 17.2 & 28.6 & 9.8 \\
\hline $80-89$ & 6.5 & 11.4 & 3.4 \\
\hline $90-99$ & 3.6 & 6.8 & 1.6 \\
\hline $100-109$ & 1.0 & 1.1 & 1.0 \\
\hline$\geq 110$ & 0.2 & 0.1 & 0.3 \\
\hline 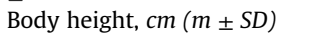 & $166.2 \pm 9.9$ & $174.1 \pm 8.3$ & $161.1 \pm 7.1$ \\
\hline range & $132-201$ & $138-201$ & $132-193$ \\
\hline \multicolumn{4}{|l|}{ Body height-class, $\mathrm{cm}(\%)$} \\
\hline$\leq 160$ & 32.6 & 6.2 & 49.7 \\
\hline$\overline{161-170}$ & 35.9 & 27.0 & 41.7 \\
\hline $171-180$ & 23.0 & 45.7 & 8.2 \\
\hline$>180$ & 8.5 & 21.1 & 0.4 \\
\hline BMI, $\mathrm{kg} / \mathrm{m}^{2}(m \pm S D)$ & $22.7 \pm 4.2$ & $22.9 \pm 3.7$ & $22.5 \pm 4.6$ \\
\hline range & $10.5-46.8$ & $13.5-39.3$ & $10.5-46.8$ \\
\hline \multicolumn{4}{|l|}{ BMI-class, $\mathrm{kg} / \mathrm{m}^{2}(\%)$} \\
\hline$\leq 15.0$ & 1.6 & 1.4 & 1.8 \\
\hline $15.1-18.5$ & 12.6 & 9.0 & 15.0 \\
\hline $18.6-25.0$ & 61.9 & 64.6 & 60.2 \\
\hline $25.1-30.0$ & 18.2 & 21.2 & 16.3 \\
\hline$>30$ & 5.6 & 3.9 & 6.7 \\
\hline \multicolumn{4}{|l|}{ SBS type (\%) } \\
\hline$S B S-J$ & 60.0 & 58.1 & 61.1 \\
\hline SBS-JC & 30.9 & 32.4 & 30.0 \\
\hline SBS-JIC & 9.1 & 9.5 & 8.9 \\
\hline \multicolumn{4}{|l|}{ Underlying disease (\%) } \\
\hline Adhesion & 2.3 & 2.0 & 2.5 \\
\hline Cancer & 1.3 & 1.9 & 0.9 \\
\hline CIPO & 2.2 & 2.3 & 2.2 \\
\hline Crohn's disease & 27.1 & 28.5 & 26.3 \\
\hline Mesenteric Ischemia & 26.7 & 30.8 & 24.1 \\
\hline Polyposis-Gardner's & 2.2 & 2.4 & 2.1 \\
\hline Radiation enteritis & 6.3 & 1.9 & 9.1 \\
\hline Surgical complications & 16.7 & 14.8 & 18.0 \\
\hline Trauma & 1.5 & 2.2 & 1.1 \\
\hline Ulcerative colitis & 1.3 & 1.8 & 1.0 \\
\hline Volvulus & 3.7 & 4.2 & 3.3 \\
\hline Other & 3.1 & 2.6 & 3.5 \\
\hline Not reported & 5.5 & 4.7 & 6.0 \\
\hline \multicolumn{4}{|l|}{ IVS } \\
\hline Duration, $m o(m \pm S D)$ & $65.2 \pm 77.4$ & $65.7 \pm 81.2$ & $64.9 \pm 75.0$ \\
\hline range & $0-474.0$ & $0-474.0$ & $0-463.1$ \\
\hline \multicolumn{4}{|l|}{ Duration-class, yr (\%) } \\
\hline$\leq 1$ & 22.8 & 25.0 & 21.4 \\
\hline $1.1-3$ & 26.0 & 24.4 & 27.0 \\
\hline $3.1-10$ & 35.4 & 35.0 & 35.4 \\
\hline$>10$ & 15.8 & 15.6 & 16.2 \\
\hline \multicolumn{4}{|l|}{ Infusion } \\
\hline Days/week, $n .(m \pm S D)$ & $5.8 \pm 1.6$ & $5.9 \pm 1.5$ & $5.8 \pm 1.6$ \\
\hline range & $1-7$ & $1-7$ & $1-7$ \\
\hline \multicolumn{4}{|l|}{ Days/week-class (\%). } \\
\hline$<3$ & 3.1 & 2.9 & 3.3 \\
\hline $3-4$ & 19.8 & 19.1 & 20.3 \\
\hline $5-6$ & 20.3 & 19.4 & 20.8 \\
\hline 7 & 56.8 & 58.7 & 55.5 \\
\hline Volume $\mathrm{mL} /$ week, $(m \pm S D)$ & $13,585 \pm 7668$ & $14,580 \pm 8096$ & $12,777 \pm 7296$ \\
\hline Range & $572-52,800$ & $572-52,800$ & $750-38,500$ \\
\hline Volume $m L /$ day $(m \pm S D)$ & $1926 \pm 1095$ & $2083 \pm 1157$ & $1825 \pm 1042$ \\
\hline Range & $82-7543$ & $82-7543$ & $107-5500$ \\
\hline Volume, $\mathrm{mL} / \mathrm{kg} /$ day, $(m \pm S D)$ & $31.8 \pm 19.0$ & $31.0 \pm 18.6$ & $32.3 \pm 19.3$ \\
\hline
\end{tabular}


Table 1 (continued)

\begin{tabular}{|c|c|c|c|}
\hline & Total n. 1880 & Males n. 738 & Females n. 1142 \\
\hline Range & $0.9-142.2$ & $0.9-142.2$ & $1.3-115.4$ \\
\hline Energy, $\mathrm{kcal} /$ week $(m \pm S D)$ & $7074 \pm 4560$ & $8221 \pm 4842$ & $6333 \pm 4208$ \\
\hline Range & $0-23,803$ & $0-23803$ & $0-21021$ \\
\hline Energy, kcal/day $(m \pm S D)$ & $1011 \pm 651$ & $1174 \pm 692$ & $905 \pm 601$ \\
\hline Range & $0-3400$ & $0-3400$ & $0-3003$ \\
\hline Energy, $\mathrm{kcal} / \mathrm{kg} / \mathrm{day},(m \pm S D)$ & $17.0 \pm 11.5$ & $17.8 \pm 11.2$ & $16.5 \pm 11.7$ \\
\hline Range & $0-74.7$ & $0-55.6$ & $0-74.7$ \\
\hline \multicolumn{4}{|c|}{ IF-class, volume/day of infusion (\%) } \\
\hline FE1 $(\leq 1 L)$ & 7.0 & 4.9 & 8.4 \\
\hline FE2 $(1-2 L)$ & 2.7 & 2.8 & 2.5 \\
\hline FE3 $(2-3 L)$ & 0.6 & 0.8 & 0.5 \\
\hline FE4 $(>3 L)$ & 0.4 & 0.4 & 0.4 \\
\hline$P N 1(\leq 1 L)$ & 16.3 & 12.6 & 18.7 \\
\hline PN2 $(1-2 L)$ & 35.4 & 36.4 & 34.8 \\
\hline PN3 $(2-3 L)$ & 23.9 & 25.9 & 22.6 \\
\hline PN4 $(>3 L)$ & 13.7 & 16.1 & 12.2 \\
\hline
\end{tabular}

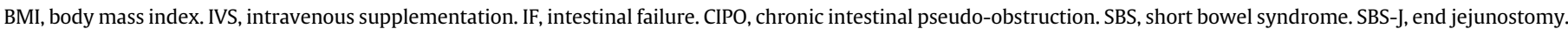

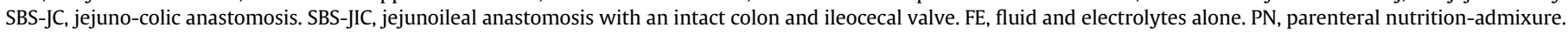

The three cohorts were similar for gender, age and BMI categories. Females were $61.9 \%, 58.9 \%$ and $59.3 \%$, and age was lower than 50 years in $31.8 \%, 28.0 \%$ and $37.2 \%$ of the SBS-J, SBS-JC and SBSJIC cohorts, respectively. BMI was below normal in 13\%, $15.9 \%, 17.1 \%$, within the normal range in $60.7 \%, 63.6 \%, 64.1 \%$, and above normal in $26.3 \%, 20.5 \%, 18.5 \%$, of the SBS-J, SBS-JC and SBS-JIC cohorts, respectively.

Differences were observed in the underlying diseases and IVS characteristics (Fig. 1). CD was more frequent in SBS-J, and MI prevailed in SBS-JC and SBS-JIC. The duration of IVS was shorter in SBS-J (less than 3 years in more than one-half), whereas it was
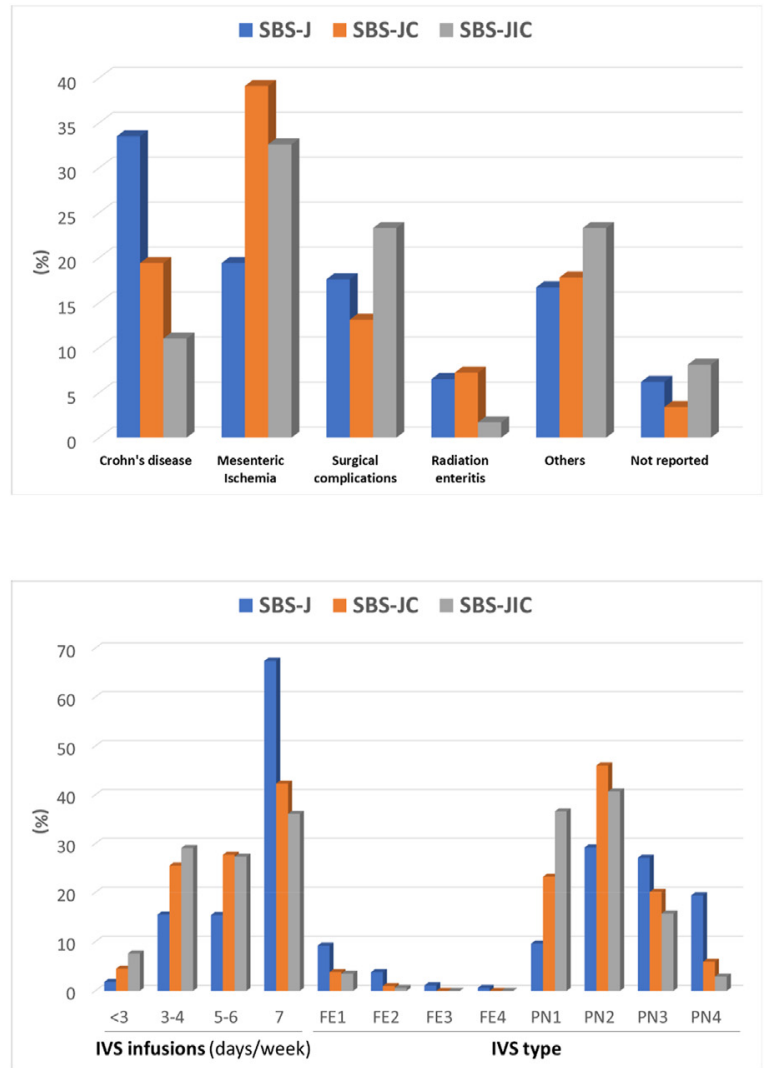

longer than 3 years in $61.4 \%$ of SBS-JC and in $52.3 \%$ of SBS-JIC. The number of days of IVS infusion per week and the percentage of patients requiring the FE type of IVS were greater in the SBS-J group. The volume of IVS was also higher in SBS-J than in SBS-JC and SBS-JIC, whereas no differences between the SBS types were observed in IVS energy.

\subsection{SBS patient cohorts in the different countries}

Country-based data are shown in Fig. 2 and are extensively reported in supplementary table 2 .
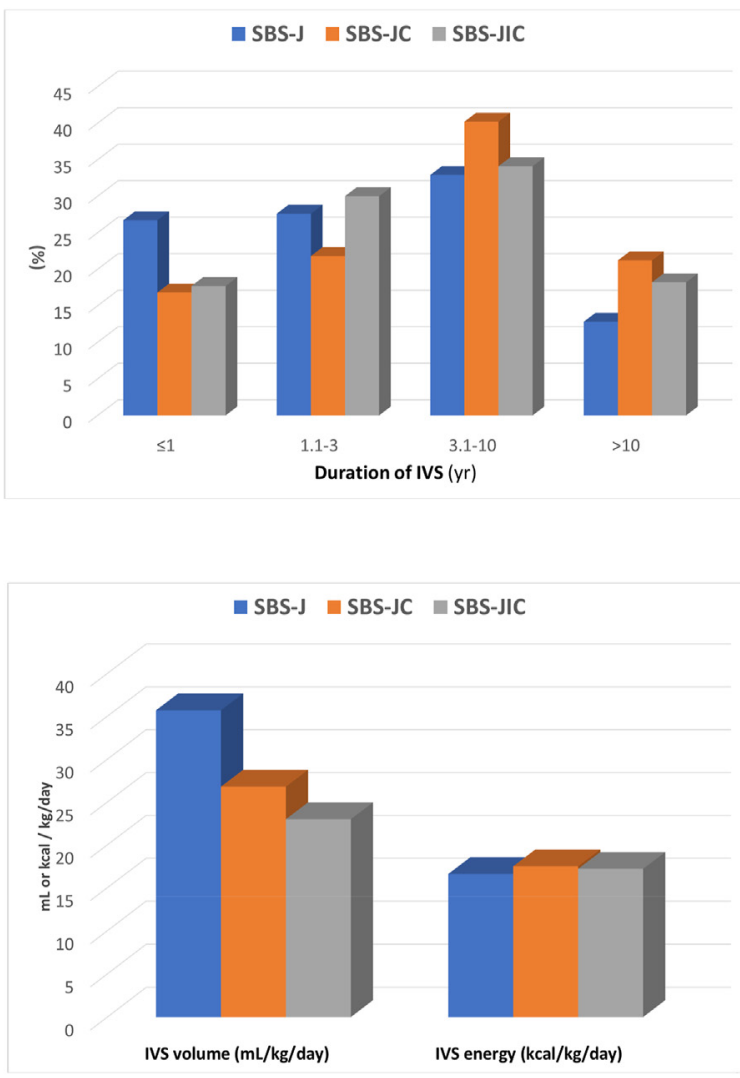

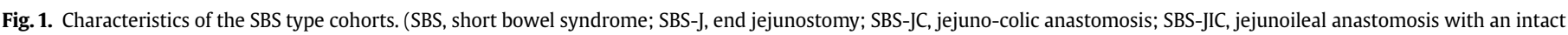
colon and ileocecal valve; IVS, intravenous supplementation). 
A)

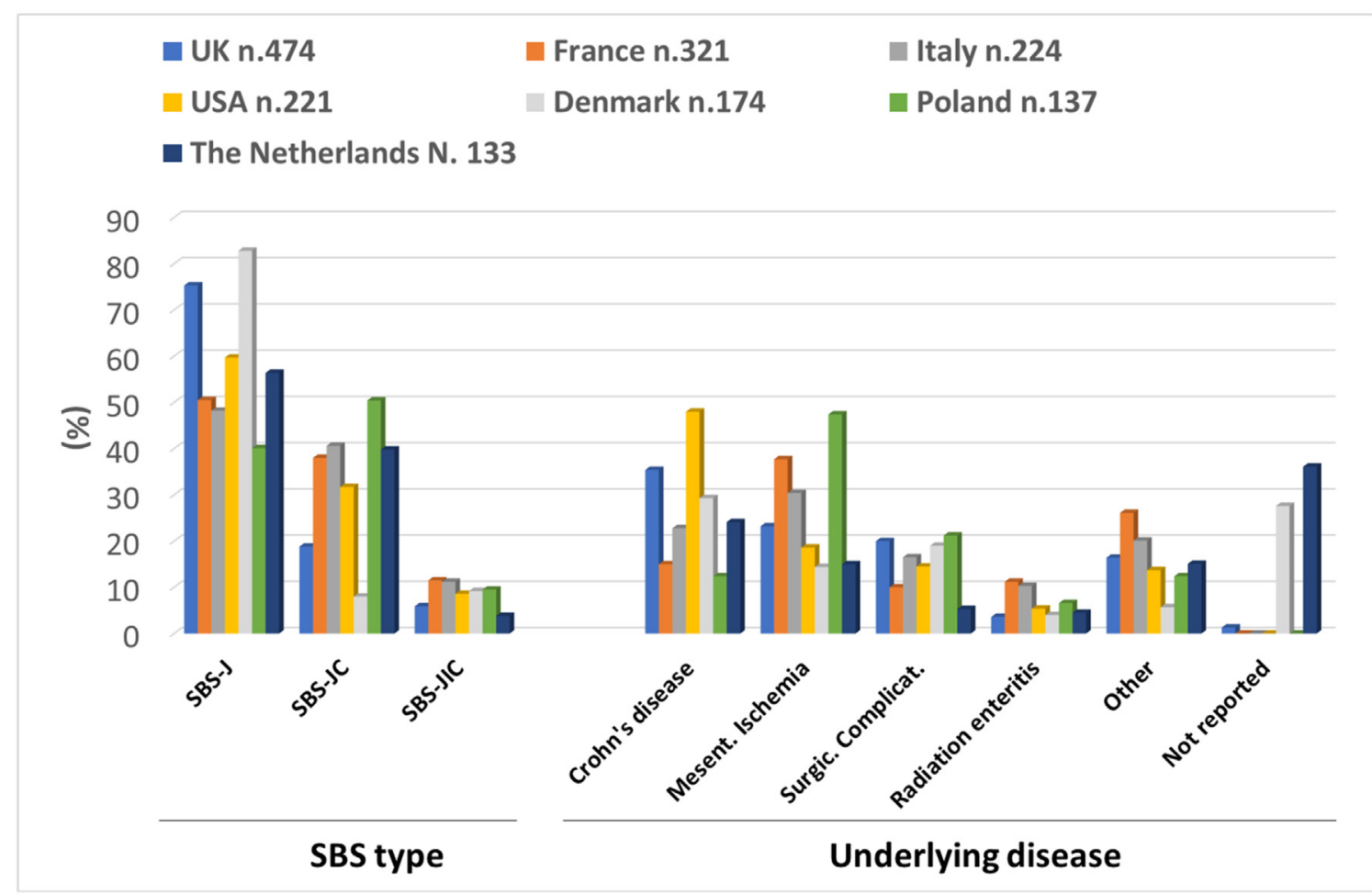

B)

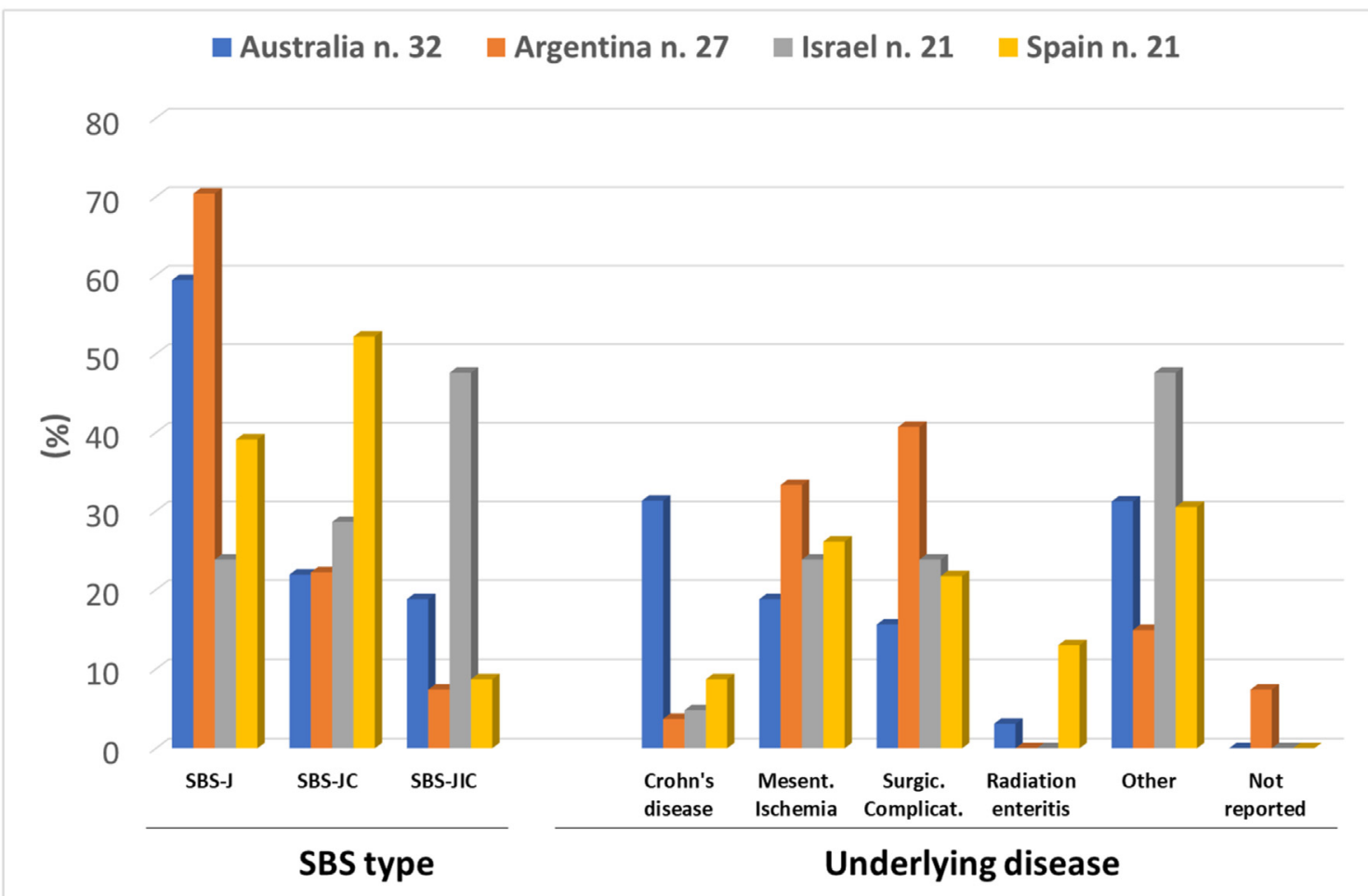

Fig. 2. SBS patient cohorts in the different countries. (SBS, short bowel syndrome; SBS-J, end jejunostomy; SBS-JC, jejuno-colic anastomosis; SBS-JIC, jejunoileal anastomosis with an intact colon and ileocecal valve). 
In the 7 countries that included more than 100 patients each: females comprised greater than $50 \%$ of patients in all cohorts; France and USA reported the youngest cohorts, with $36.7 \%$ and $38.0 \%$ of patients aged less than 50 years, respectively; malnutrition $\left(\mathrm{BMI}<18.5 \mathrm{~kg} / \mathrm{m}^{2}\right)$ was more frequent in the French (21.9\%), Danish (23.8\%) and Polish (18.9\%) groups and overweight and obesity in the British group (34.4\%).

SBS-J represented $75 \%$ or more of patients in the UK and Denmark cohorts, and $50-60 \%$ of patients in the other countries, with the exception of Poland where it comprised $40 \%$ and where SBS-JC was the most frequent SBS type. CD was the main underlying disease in the UK, USA, Denmark and The Netherlands, whereas MI prevailed in France, Italy and Poland (Fig. 2A).

The shortest durations of IVS were reported in USA and Poland, where it was less than 3 years in $51.5 \%$ and $60.6 \%$ of patients, respectively; in four countries (USA, Denmark, Poland and The Netherlands), $70 \%$ or more patients received the IVS infusion 7 days per week. The greatest percentages of FE type of IVS were observed in France (20.5\%) and The Netherlands (21.3\%); no patient received the FE type in Poland.

Differences in type of SBS, underlying diseases, duration of IVS, number of days of IVS infusion per week and type of IVS were observed among the cohorts of the 4 countries which included 20 to 40 patients (Australia, Argentina, Spain, and Israel) and are demonstrated in Fig. 2B. Differences were also apparent among the countries which each included less than 20 patients (Supplementary table 1).

\section{Discussion}

The data represent the largest study to-date demonstrating that clinically-relevant differences exist in SBS-IF types and underlying diseases between different countries, collected from multiple international centres at the same time point. Notably, the dataset also confirms differences between genders and SBS types, while also providing a global picture of the characteristics of SBS-IF cohorts within different countries of origin. In the whole group, the female to male ratio was 2:1, SBS-J was the prevalent type of SBS, MI and $\mathrm{CD}$ were the more frequent underlying diseases, BMI was normal in most patients, IVS dependency was longer than 3 years in around one-half of the cases, IVS was infused 5 days or more per week in three-fourth and consisted of FE in around 10\%.

The highest percentage of females was also observed in all three SBS-types, as well as in the individual country cohorts, a finding supported by older investigations noting that normal human small intestinal length tends to be shorter in women [1]. An individual study reported a small bowel length range of 488-785 (mean 637) $\mathrm{cm}$ in men and of 335-716 (mean 592) $\mathrm{cm}$ in women [10]. Furthermore, a previous review of eight studies demonstrated a correlation between intestinal length (range 275-850 cm) and body height in men [11]. Thus, since we also and predictably observed a shorter body height in females, a shorter baseline bowel length in women could be an explanation for the higher frequency of SBS noted in our study in females. However, recent data on the bowel length in healthy adults measured by modern technologies are lacking. Moreover, our gender cohorts did not differ in any other characteristics, except a higher frequency of radiation enteritis in females, probably due to the more frequent use of radio-therapy in treating gynaecological malignancy.

The main differences among the SBS-type cohorts were those between the SBS-J and both SBS-JC and SBS-JIC. In SBS-J, CD, as the underlying disease, was twice and thrice more frequent than in SBS-JC and SBS-JIC, respectively, whereas the opposite was observed for MI. In parallel, we also noted a higher percentage of patients with SBS-J having an IVS duration shorter than 1 year and a lower percentage on very long term ( $>10$ years) IVS. The reasons for these findings are not directly evident in the present survey. Possible explanations could be the higher 1-year risk of death on HPN observed in patients with SBS-J [9] and/or the creation of an SBS-J as a transient solution for safety reasons during abdominal surgery, with subsequent restoration of intestinal continuity leading these individuals ultimately weaning off IVS [12]. The observed highest number of days of IVS per week, of IVS volume and of FE type in SBS-J are, of course, consistent with the greatest intestinal fluid and electrolyte losses in SBS without a colon in continuity $[1,2,4]$.

The patient cohorts included by countries differed significantly in a number of characteristics. CD was the main underlying disease in UK (35.4\%), USA (48.0\%), Denmark (29.3\%) and Poland (24.1\%). The same countries had the highest percentages of SBS-J (UK, 75.3\%; USA, 59.7\%; Denmark, 82.8\%; Poland, 56.4\%), thus confirming the association between the underlying disease and type of SBS. The difference in $\mathrm{CD}$ percentages is perhaps most relevant; assuming that the prevalence and the severity of $C D$ were the same in all the countries participating in the survey, the differences in the percentages of patients with SBS-IF due to CD could be determined by the disease treatment strategies adopted by different countries and/or centers contributing to the ESPEN database. Insight into the relative types of SBS and underlying diseases are clearly important for planning national healthcare system resources for both HPN provision and SBS therapy. Indeed, as outlined earlier, IVS needs of patients with SBS-J are greater than those of the other SBS types with colon in continuity [3]; greater IVS needs, being surrogate marker of CIF severity, are, in turn, associated with poorer outcome and greater risk of major IF/HPN-related complications [9]. Furthermore, highest IVS volume requires more days of IVS infusion per week and more hours of infusion per day, with a resultant negative impact on a patient's quality of life [13].

Therapy for SBS aims to improve the function of the remnant bowel and to reduce the need of IVS with possible weaning from HPN, thus decreasing the risk of complications and outcome and quality of life [4]. The most recent advance in pharmacological treatment for SBS-IF has been the approval of teduglutide, an analogue of glucagon like peptide-2, by the European Medical Agency and by the Food and Drug Administration. Teduglutide enhances post-resection intestinal adaptation in SBS by stimulating intestinal mucosal growth and slowing gastrointestinal transit time, thus increasing intestinal absorption and decreasing intestinal losses in patients with SBS [14-18]. The response to treatment with teduglutide, with the consequent reduction in IVS requirements, has been shown to be greater in SBS-IF with the highest baseline IVS volume requirement $[19,20]$ and in those with SBS-J [19]. Furthermore, the decision to use teduglutide in SBS-IF due to $C D$, the main cause of SBS-J in the present study, must take into account associated therapeutic precautions related to the presence of active disease, fistulas, strictures and the concomitant use biologic drugs $[5,21]$. It is also important to recognize that other differences among countries noted in IVS characteristics may have not only been determined by SBS-types or underlying disease, but also by the established variation that is known to exist in the approach adopted by individual countries and centres to the organization and management of CIF and HPN $[22,23]$. This variability can influence the epidemiology of both CIF and HPN provision. The lack of standardization of regulations and procedures for CIF is probably unique in the panorama of all chronic organ failures and requires resolution [24].

The strength of the study is reflected by its international multicentre structure and by the study population, which is the largest cohort of SBS-IF patients ever enrolled in a single survey. Limitations to be considered are the voluntary participation of the HPN 
centres as well as the date of data collection, that was 6 years before this sub-analysis. These limitations may imply that, notwithstanding the fact that the participating HPN centres were among the largest of their countries, they may not be representative of the entire country's experience, and that the epidemiology of SBS-IF in 2021 may differ from that of 2016, even though a significant change in SBS-IF pathogenesis seems unlikely within a 6-year timeframe. In conclusion, the analysis of the SBS-IF cohorts of patients included in the ESPEN CIF database provides useful information for planning and managing both clinical activity and research studies on SBS. Overall, SBS-IF was twice as frequent in females and SBS-J was the most frequent type of SBS. Significant variations among countries were observed in underlying diseases and IVS characteristics, which may be determined by differences in clinical practice, as well as in service organization for managing CIF and HPN.

\section{Acknowledgements}

Contributing coordinators and centers by country

\section{Argentina}

Adriana N. Crivelli, Hector Solar Muñiz; Hospital Universitario Fundacion Favaloro, Buenos Aires

\section{Australia}

Brooke R. Chapman; Austin Health, Melbourne Lynn Jones; Royal Prince Alfred Hospital, Sydney Margie O'Callaghan; Flinders Medical Centre, Adelaide Emma Osland, Ruth Hodgson, Siobhan Wallin, Kay Lasenby; Royal Brisbane and Women's Hospital, Herston

\section{Belgium}

Andre Van Gossum: Hôpital Erasme, Brussels

Brazil:

Andre Dong Won Lee; Hospital das Clinicas da Faculdade de Medicina da Universidade de São Paulo, São Paulo

\section{Bulgaria}

Maryana Doitchinova-Simeonova; Bulgarian Executive Agency

of Transplantation, Sofia

\section{Croatia}

Zeljko Krznaric; University Hospital Centre Zagreb, Zagreb

Denmark

Henrik Højgaard Rasmussen; Center for Nutrition and Bowel

Disease, Aalborg University Hospital, Aalborg

Chrisoffer Brandt, Palle B. Jeppesen; Rigshospitalet, Copenhagen France

Cecile Chambrier; Hospices Civils de Lyon, Centre Hospitalier Lyon Sud, Lyon

Francisca Joly, Vanessa Boehm, Julie Bataille, Lore Billiauws; Beaujon Hospital, Clichy

Florian Poullenot; CHU de Bordeaux, Hôpital Haut-Lévêque, Pessac

Stéphane M. Schneider; CHU Archet, Nice

David Seguy; CHRU de Lille, Lille

Ronan Thibault; Nutrition unit, CHU Rennes, Nutrition Metabolisms and Cancer institute, NuMeCan, INRA, INSERM, Université Rennes, Rennes

\section{Germany}

Jann Arends; Tumor Biology Center, Freiburg

\section{Hungary}

Laszlo Czako, Tomas Molnar, Mihaly Zsilak-Urban; University of Szeged, Szeged

Ferenc Izbéki; Szent György Teaching Hospital of County Fejér,

Székesfehérvár

Peter Sahin, Gábor Udvarhelyi; St. Imre Hospital, Budapest

Eszter Schafer; Magyar Honvedseg Egészségügyi Központ

(MHEK), Budapest

Israel
Miriam Theilla; Rabin Medical Center, Petach Tikva

Italy

Anna Simona Sasdelli, Giorgia Brillanti, Elena Nardi, Loris Pironi;

IRCCS S. Orsola University Hospital, Bologna

Umberto Aimasso, Merlo F. Dario; Città della Salute e della Scienza, Torino

Valentino Bertasi, Luisa Masconale; ULSS 22 Ospedale Orlandi, Bussolengo

Francesco W. Guglielmi, Nunzia Regano; San Nicola Pellegrino Hospital, Trani

Paolo Orlandoni; Nutrizione Clinica-Centro di Riferimento Regionale NAD, IRCCS-INRCA, Ancona, Italy

Santarpia Lidia, Lucia Alfonsi; Federico II University, Italy

Corrado Spaggiari; AUSL di Parma, Parma

Marina Taus, Debora Busni; Ospedali Riuniti, Ancona

Lithuania

Gintautas Kekstas; Vilnius University Hospital Santariskiu Clinics, Vilnius

México

Aurora E. Serralde-Zúñiga; Instituto Nacional de Ciencias Médicas y Nutrición Salvador Zubirán, México City

New Zealand

Lyn Gillanders; Auckland City Hospital, Auckland

\section{Poland}

Marek Kunecki; M. Pirogow Hospital, Lodz

Przemysław Matras; Medical University of Lublin, Lublin

Konrad Matysiak; H.Święcicki University Hospital, Poznań

Kinga Szczepanek; Stanley Dudrick's Memorial Hospital, Skawina

Anna Zmarzly; J. Gromkowski City Hospital, Wroclaw

Slovenia

Nada Rotovnik Kozjek; Institute of Oncology, Ljubljana

\section{Spain}

Marta Bueno; Hospital Universitario Arnau de Vilanova, Lleida

Cristina Cuerda; Hospital General Universitario Gregorio Marañon, Madrid

Carmen Garde; Hospital Universitario Donostia, San Sebastian Nuria M. Virgili; Hospital Universitari de Bellvitge, Barcelona

Gabriel Olveira; IBIMA, Hospital Regional Universitario de Málaga, Universidad de Málaga, Málaga

$\mathrm{M}^{\mathrm{a}}$ Estrella Petrina Jáuregui; Complejo Hospitalario de Navarra, Pamplona

Alejandro Sanz-Paris; Miguel Servet Hospital, Zaragoza

José P. Suárez-Llanos; Hospital Universitario Nuestra Señora de Candelaria, Santa Cruz de Tenerife

Ana Zugasti Murillo; Hospital Virgen del Camino, Pamplona

Sweden

Lars Ellegard; Sahlgrenska University Hospital, Gothenburg

\section{The Netherlands}

Mireille Serlie, Cora Jonker; Academic Medical Center, Amsterdam Geert Wanten; Radboud University Medical Center, Nijmegen United Kingdom

Sheldon C. Cooper; University Hospitals Birmingham NHS

Foundation Trust, Birmingham

Joanne Daniels; Nottingham University Hospital NHS Trust, Nottingham

Simona Di Caro, Niamh Keane, Pinal Patel; University College Hospital, London

Alastair Forbes; Norfolk and Norwich University Hospital, Norwich

Sarah-Jane Nelson Hughes; Regional Intestinal Failure Service,

Belfast Health and Social Care Trust, Northern Ireland

Amelia Jukes, Rachel Lloyd; University Hospital of Wales, Cardiff

Simon Lal, Arun Abraham, Gerda Garside, Michael Taylor; Salford Royal NHS Foundation Trust, Salford 
Jian Wu, Trevor Smith, Charlotte Pither, Michael Stroud; University Hospital Southampton NHS Foundation Trust, Southampton Nicola Wyer, Reena Parmar, Nicola Burch; University Hospital, Coventry

Sarah Zeraschi; Leeds Teaching Hospitals NHS Trust, Leeds

\section{United States of America}

Charlene Compher; Hospital of the University of Pennsylvania,

Philadelphia, PA

Manpreet Mundi; Mayo Clinic College of Medicine, Rochester, MN

Denise Jezerski, Ezra Steiger; Cleveland Clinic Foundation, Cleveland, $\mathrm{OH}$

ESPEN CIF database managers and statisticians: Elena Nardi, Department of Experimental, Diagnostic and Specialty Medicine; Giorgia Brillanti, Department of Medical and Surgical Sciences; University of Bologna, Italy.

\section{Funding source}

The project of the ESPEN database for Chronic Intestinal Failure was promoted by the ESPEN Executive Committee in 2013, was approved by the ESPEN Council and was supported by an ESPEN grant.

\section{Statement of authorship}

LP devised the study protocol, collected the data, analyzed the results and drafted the manuscript. YN contributed to the study protocol and revised the final manuscript. The Home Artificial Nutrition \& Chronic Intestinal Failure Special Interest Group of ESPEN discussed and approved the protocol study, discussed the results and reviewed the manuscript before submission. According to the authorship rules described in the protocol study, coordinators of the participating centers were considered coauthors of the study and received the manuscript upon submission. All authors approved the final version of the manuscript before submission.

\section{Declaration of competing interest}

None declared.

\section{Appendix A. Supplementary data}

Supplementary data to this article can be found online at https://doi.org/10.1016/j.clnesp.2021.07.004.

\section{References}

[1] Nightingale J, Woodward JM, Bowel Small, Nutrition. Committee of the British Society of Gastroenterology. Guidelines for management of patients with a short bowel. Gut 2006 Aug;55(Suppl 4):iv1-12.

[2] Pironi L. Definitions of intestinal failure and the short bowel syndrome. Best Pract Res Clin Gastroenterol 2016 Apr;30(2):173-85.

[3] Pironi L, Konrad D, Brandt C, Joly F, Wanten G, Agostini F, et al. Clinical classification of adult patients with chronic intestinal failure due to benign disease: an international multicenter cross-sectional survey. Clin Nutr 2018;37(2):728-38.

[4] Pironi L, Arends J, Bozzetti F, Cuerda C, Gillanders L, Jeppesen PB, et al. ESPEN guidelines on chronic intestinal failure in adults. Clin Nutr 2016 Apr;35(2): 247-307.

[5] Bond A, Taylor M, Abraham A, Teubner A, Soop M, Carlson G, et al. Examining the pathophysiology of short bowel syndrome and glucagon-like peptide 2 analogue suitability in chronic intestinal failure: experience from a national intestinal failure unit. Eur J Clin Nutr 2019 May;73(5):751-6.

[6] Brandt CF, Tribler S, Hvistendahl M, Staun M, Brøbech P, Jeppesen PB. Singlecenter, adult chronic intestinal failure cohort analyzed according to the ESPEN-endorsed recommendations, definitions, and classifications. J Parenter Enter Nutr 2017:41(4):566-74.

[7] Amiot A, Messing B, Corcos O, Panis Y, Joly F. Determinants of home parenteral nutrition dependence and survival of 268 patients with non-malignant short bowel syndrome. Clin Nutr Edinb Scotl 2013 Jun;32(3):368-74.

[8] Pironi L, Sasdelli AS, Venerito FM, Musio A, Pazzeschi C, Guidetti M. Candidacy of adult patients with short bowel syndrome for treatment with glucagon-like peptide-2 analogues: a systematic analysis of a single centre cohort. 2021. p. 85-6. https://authors.elsevier.com/sd/article/S0261-5614(21).

[9] Pironi L, Steiger E, Joly F, Wanten GJA, Chambrier C, Aimasso U, et al. Intravenous supplementation type and volume are associated with 1-year outcome and major complications in patients with chronic intestinal failure. Gut 2020 Oct;69(10):1787-95.

[10] Underhill BM. Intestinal length in man. Br Med J 1955 Nov 19;2(4950): 1243-6. https://doi.org/10.1136/bmj.2.4950.1243. PMID: 13269841; PMCID: PMC1981305.

[11] Weaver LT, Austin S, Cole TJ. Small intestinal length: a factor essential for gut adaptation. Gut 1991 Nov;32(11):1321-3. https://doi.org/10.1136/ gut.32.11.1321. PMID: 1752463; PMCID: PMC1379160.

[12] Fuglsang KA, Brandt CF, Scheike T, Jeppesen PB. Differences in methodology impact estimates of survival and dependence on home parenteral support of patients with nonmalignant short bowel syndrome. Am J Clin Nutr 2020 Jan 1;111(1):161-9. https://oi.org/10.1093/ajcn/nqz242. PMID: 31562502.

[13] Baxter JP, Fayers PM, Bozzetti F, Kelly D, Joly F, Wanten G, et al. Home artificial nutrition and chronic intestinal failure special interest group of the European society for clinical nutrition and metabolism (ESPEN). An international study of the quality of life of adult patients treated with home parenteral nutrition. Clin Nutr 2019 Aug;38(4):1788-96. https://doi.org/10.1016/j.clnu.2018.07. 024. Epub 2018 Aug 6. PMID: 30115461.

[14] Jeppesen PB. Teduglutide for the treatment of short bowel syndrome. Drugs Today 2013 Oct;49(10):599-614. https://doi.org/10.1358/dot.2013.49.10. 2017025. PMID: 24191254.

[15] Daoud DC, Joly F. The new place of enterohormones in intestinal failure. Curr Opin Clin Nutr Metab Care 2020 Sep;23(5):344-9. https://doi.org/10.1097/ MCO.0000000000000672. PMID: 32618723.

[16] Billiauws L, Joly F. Emerging treatments for short bowel syndrome in adult patients. Expet Rev Gastroenterol Hepatol 2019 Mar;13(3):241-6. https:// doi.org/10.1080/17474124.2019.1569514. Epub 2019 Feb 7. PMID: 30791759.

[17] Pironi L. Translation of evidence into practice with teduglutide in the management of adults with intestinal failure due to short-bowel syndrome: a review of recent literature. J Parenter Enter Nutr 2020 Aug;44(6):968-78. https://doi.org/10.1002/jpen.1757. Epub 2019 Dec 4. PMID: 31802516.

[18] Jeppesen PB. The long road to the development of effective therapies for the short gut syndrome: a personal perspective. Dig Dis Sci 2019 Oct:64(10): 2717-35. https://doi.org/10.1007/s10620-019-05779-0. PMID: 31410752.

[19] Jeppesen PB, Gabe SM, Seidner DL, Lee HM, Olivier C. Factors associated with response to teduglutide in patients with short-bowel syndrome and intestinal failure. Gastroenterology 2018 Mar;154(4):874-85. https://doi.org/10.1053/ j.gastro.2017.11.023. Epub 2017 Nov 22. PMID: 29174926.

[20] Joly F, Seguy D, Nuzzo A, Chambrier C, Beau P, Poullenot F, et al. Six-month outcomes of teduglutide treatment in adult patients with short bowel syndrome with chronic intestinal failure: a real-world French observational cohort study. Clin Nutr 2020 Sep;39(9):2856-62. https://doi.org/10.1016/ j.clnu.2019.12.019. Epub 2019 Dec 23. PMID: 31932048.

[21] Pironi L, Sasdelli AS, Venerito FM, Musio A, Pazzeschi C, Guidetti M. Candidacy of adult patients with short bowel syndrome for treatment with glucagon-like peptide-2 analogues: a systematic analysis of a single centre cohort. Clin Nutr 2021 Jun;40(6):4065-74. https://doi.org/10.1016/j.clnu.2021.02.011. Epub ahead of print. PMID: 33637328

[22] Wengler A, Micklewright A, Hebuterne X, Bozzetti F, Pertkiewicz M, Moreno J, et al. Monitoring of patients on home parenteral nutrition (HPN) in Europe: a questionnaire based study on monitoring practice in 42 centres. Clin Nutr 2006;25:693-e700.

[23] Pironi L, Steiger E, Brandt C, Joly F, Wanten G, Chambrier C, et al. Home artificial nutrition and chronic intestinal failure special interest group of ESPEN; European society for clinical nutrition and metabolism. Home parenteral nutrition provision modalities for chronic intestinal failure in adult patients: an international survey. Clin Nutr 2020 Feb;39(2):585-91. https:// doi.org/10.1016/j.clnu.2019.03.010. Epub 2019 Mar 25. PMID: 30992207.

[24] Pironi L, Boeykens K, Bozzetti F, Joly F, Klek S, Lal S, et al. ESPEN guideline on home parenteral nutrition. Clin Nutr 2020 Jun;39(6):1645-66. https://doi.org/ 10.1016/j.clnu.2020.03.005. Epub 2020 Apr 18. PMID: 32359933. 